\title{
Dapp Blockchain en el alquiler tradicional de Viviendas en Lima
}

\author{
Dapp Blockchain in the traditional rental of Housing in Lima \\ Dapp Blockchain no aluguel tradicional de Habitação em Lima
}

\section{ARTÍCULO GENERAL}

\author{
César Loo Gil \\ https://orcid.org/0000-0001-8396-5972 \\ cesarloo@biofab.com.pe \\ Científico Investigador de BioFab Inc, Lima - Perú
}

Recibido 01 de Diciembre 2020 | Arbitrado y aceptado 26 de Diciembte 2020 | Publicado el 01 de Febrero 2021

\section{RESUMEN}

La tecnología Blockchain ha permitido el registro de información, valores y activos en cualquiera de sus formas sin la ayuda de ningún intermediario se considera una de las tecnologías más innovadoras del último tiempo con la función de cambiar sistemas transaccionales otorgando estabilidad, transparencia, descentralización y participación a medida que concede poder a los usuarios, además tiene la función de mejorar procesos $y$ minimizar los precios de las transacciones. Las Dapp blockchain tienen el potencial para ser usadas en el alquiler tradicional de viviendas en Lima, por consiguiente, es fundamental comprender como impacta y favorece a las organizaciones y sus modelos de negocios. Por todo ello, el presente artículo de investigación se propuso reflexionar sobre entorno del alquiler tradicional de viviendas usando la Tecnología Dapp Blockchain, sus usos potenciales, impactos y beneficios para este modelo de negocio. Este tipo de empresas deben buscar formas de reducir las amenazas de la tecnología ya sea incorporándola o ajustándolo al modelo de negocio.

Palabras clave: Dapp Blockchain, tecnología, alquiler de viviendas

\section{ABSTRACT}

Introduction: COVID-19 is an infectious disease caused by the new coronavirus, its peculiarity is to transmit rapidly from person to person, through droplets, which are expelled through the mouth or nose when the person breathes in or out the oxygen in the air, reason for which it is essential to apply the measures of hand washing with soap and water, the use of masks, physical and social distancing in the inhabitants. The lack of knowledge about the prevention of COVID19 has caused the accelerated increase in cases, causing it to occur, hospitalizations, deaths, in addition to expenses and problems of discrimination against those who are carriers of the disease.

Objetive: Determine the influence of the Educational Intervention to prevent COVID19 in the inhabitants of the "Amplification La Victoria" Housing Association of the district of the Banda de Shilcayo 2021.

Materials and methods: It has been made a quasi-experimental study, before-after type without a control group, was carried out through an educational intervention to the inhabitants of the Housing Association of the Banda de Shilcayo district during the period from June to December 2021. The study was made up of the 150 inhabitants of the Housing Association. We worked with $100 \%$ of the population

Results: It was observed a significant increase in the level of high knowledge was observed after the educational intervention about the disease $(54 \%)$, healthy lifestyles $(96.7 \%)$ and prevention measures $(100 \%)$

Conclusions

The educational intervention was effective because a high level of knowledge was achieved in the inhabitants of the Housing Association

Key words: educational intervention; prevention, COVID-19; coronavirus.

\section{RESUMO}

Introdução: A falta de conhecimento sobre a prevenção da COVID-19 tem ocasionado o aumento acelerado de casos, ocasionando internações, óbitos, além de gastos e problemas de discriminação para os portadores da doença. O objetivo foi determinar a influência da Intervenção Educativa para prevenir o COVID-19 nos habitantes da Associação Habitacional "Amplificação La Victoria" do distrito de Banda de Shilcayo, San Martín Peru.

Métodos: Realizou-se um estudo quase experimental, do tipo antes-depois sem grupo de controlo através de uma intervenção educativa que consistiu na aplicação de oficinas educativas a cada 15 dias aos habitantes da Associação de Habitação, durante o período de junho a dezembro de $2021 \mathrm{O}$ estudo consistiu em 150 pessoas representando $100 \%$ da população adulta.

Resultado: observou-se aumento significativo do nível de conhecimento elevado após a intervenção educativa sobre a doença (54\%), estilos de vida saudáveis $(96,7 \%)$ e medidas de prevenção $(100 \%)$. mais tarde nas respostas finais ou diferenças entre antes e depois??? São conhecimentos, aptidões ou comportamentos?

Conclusões: A intervenção educativa foi eficaz porque alcançou um

alto nível de conhecimento nos habitantes da Associação de Habitação.

Palavras-chave: coronavírus, COVID-19, intervenção educativa; prevenção. 


\section{Introducción}

El ritmo de la innovación tecnológica y de los negocios se ha acelerado a lo largo

de las últimas décadas, dificultando la preparación de modelos de negocios que mantengan una productividad sostenida en la era. En particular, Internet posibilita la construcción de nuevos modelos de negocios con un alcance universal instantáneo (Teece \& Linden, 2017). Sin embargo internet carece en si misma de medios de pago, estructuras corporativas y formas de asociación que invalidan la separación del mundo digital del real (Lérida \& Pérez, 2016).

En todo el tiempo las revoluciones tecnológicas tuvieron un efecto positivo en el desarrollo, aumento y novedad tanto en el campo público como el privado, generando cambio en diversas industrias entregando nuevos productos, maneras de venta y modificación de la manera en que las organizaciones producen costo para el mercado. Sin embargo las anteriores revoluciones se podrían distinguir en la manera en la que se entrega y captura costo para los consumidores, en este sentido blockchain con sus características que le permiten no depender de un tercero de confianza promete beneficios para la sociedad, motivando el interés por la participación.

Las modalidades y oportunidades que proporciona la tecnología blockchain son distintas con una pluralidad de aplicaciones en diversas industrias que dan mejoras de procesos $\mathrm{y}$ transformaciones en la manera que se relacionan organizaciones y consumidores. Estas propiedades y utilidades han despertado el interés por examinar la tecnología, su ecosistema, efecto y beneficios para las organizaciones, con el propósito de dar al entendimiento tanto académico como empresarial.

Hasta día de hoy los obstáculos que como yo, muchos otros potenciales o actuales arrendatarios han podido encontrar son los siguientes:

- Altos honorarios de las agencias inmobiliarias.

- Altos precios de renta mensual.

- Baja oferta frente a la alta demanda de viviendas para alquilar, especialmente contratos, extractos bancarios, declaraciones de la renta... etc.).

- Dificultad para encontrar perfiles de compañeros similares. en grandes ciudades. 
- Excesiva documentación como requisito para demostrar solvencia (nóminas,

- Fianzas y depósitos que alcanzan la cuantía de cuotas de hasta 3 meses.

- Necesidad de ver el inmueble físicamente.

Estas son varias de las causas que dificultan a la mayor parte de personas que se hallan en averiguación de una casa en alquiler, que en un elevado porcentaje hablamos de personas adolescentes que, o bien no tienen la posibilidad de permitirse la compra de una casa, o que por motivos laborales se ven forzados a modificar su sitio de residencia.

El incremento de la proporción de hogares que residen en vivienda de alquiler se intensificó a partir de 2013, y se concentró en el segmento del alquiler a precio de mercado (14,8\% en 2018 , frente al $9,5 \%$ en 2005$)$, mientras que se ha producido un descenso del alquiler social (2,7\% en 2018, frente al 3,5\% de 2005). Por su parte, las cesiones gratuitas de vivienda han oscilado ligeramente en torno al 6,4\% en los últimos años. En conjunto, el número de hogares en régimen de alquiler se situaba algo por encima de los 3 millones en 2018, frente a los 2,4 millones de 2008. En este contexto, como muestra el Figura 1, lo que más destaca es el crecimiento de la proporción de vivienda en alquiler a precio de mercado.

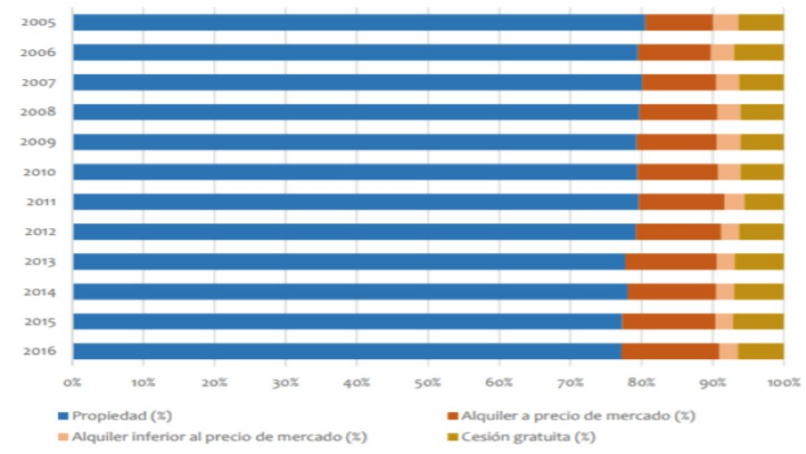

Figura 1. Hogares por régimen de tendencia (\%)

Fuente: INEI Encuesta de condiciones de vida

Estas son varias de las causas que dificultan a la mayor parte de personas que se hallan en averiguación de una casa en alquiler, que en un elevado porcentaje hablamos de 
personas adolescentes que, o bien no tienen la posibilidad de permitirse la compra de una casa, o que por motivos laborales se ven forzados a modificar su sitio de residencia.

Definimos el mercado de referencia como aquel que cubre una necesidad genérica. Para definir y representar gráficamente el mercado de referencia lo hacemos a través del modelo de Abell, quien plantea tres dimensiones para delimitar éste: el grupo de los clientes a los que se satisface, la función de los clientes que se cubren y la dimensión tecnológica utilizada para ello. Mediante este modelo se responde a las siguientes preguntas: ¿a quién se atiende?, ¿qué necesidad se satisface? y ¿cómo se satisfacen las funciones de los clientes? (Abell, 1980).

Dicho marco de referencia o campo de actividad es aquel que cubre una necesidad genérica. A continuación representamos gráficamente la empresa a través de dicho modelo mencionado anteriormente:

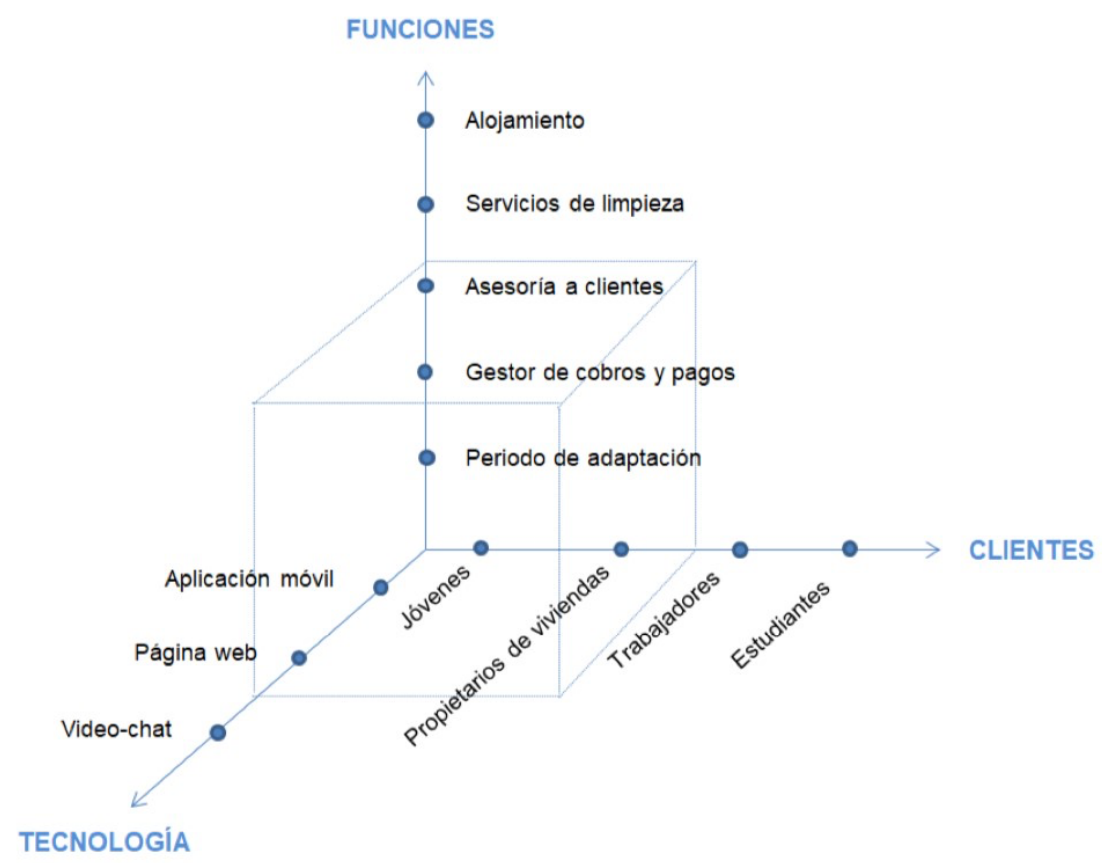

Figura 2. Necesidades genéricas

Fuente: Elaboración propia 
En el Figura de Abell, mostrado previamente, se definen sus clientes objetivos, las principales funciones que las Dapp blockchain deben ofrecer, así como la tecnología a través de la cual se ofrecen los servicios a estos mismos.

La tecnología Blockchain ha experimentando un auge importante durante los últimos años. De la misma manera que internet cambió para siempre los modelos de negocio de industrias y empresas centenarias, blockchain o «cadena de bloques» está dando lugar, desde su aparición en 2009, a un nuevo patrón económico basado en la descentralización de la confianza, en donde todos podremos intercambiar bienes y servicios sin necesidad de terceros. Esto implica un avance sin parangón que revolucionara la manera en la que organizamos nuestra vida digital. De hecho, no en vano se dice que hemos pasado del internet de la información al internet del valor (Preukschat, 2017). La tecnología blockchain se considera la invención más significativa después de Internet (Efanov \& Roschin, 2018).

\section{Metodología}

La metodología utilizada consta de búsqueda de información de paper, revistas, análisis de aplicaciones actuales y páginas web especializadas para entender su funcionamiento, el nivel de desarrollo obtenido en la actualidad y cuáles son sus principales aplicaciones. Además, se emplean análisis de modelos estratégicos acordes para analizar el atractivo de las industrias en la adopción de la tecnología y así determinar a través de modelos de análisis de negocio cuáles son las industrias en que tendrá mayor incidencia.

La búsqueda de la información para la revisión de literatura se hizo consultando las siguientes bases de datos como son Proquest, Scopus y Google Scholar para encontrar revistas del más alto nivel científico. Asimismo, cabe mencionar que para la realización de lo mencionado se emplearon también palabras clave como como Dapp Blockchain, tecnología, alquiler de viviendas.

Cada uno de estos descriptores se combinaron entre sí durante la búsqueda utilizando los operadores boléanos “and” y “or". Además, algunos de los criterios o filtros que se utilizaron para hacer muchos más específica la búsqueda y ser más precisos con 
los documentos encontrados en las diferentes bases de datos consultadas fueron: que sean artículos de revistas, y la antigüedad de la publicación que no sea mayor a 5 años.

Toda la información previa relacionada a los filtros aplicados y las especificaciones sirvieron como criterios de inclusión, es decir que sean artículos publicados en revistas científicas, que no excedan los cinco años de antigüedad, que la temática guarde relación con las variables de creatividad artística y tiempos de pandemia. Se excluyeron, en tanto, todos los documentos que no respetaran lo postulado así como aquellos que estaban incompletos o que tenían enlaces averiados.

\section{Resultados y discusión}

\subsection{Dapp blockchain}

\subsubsection{Nociones básicas}

Blockchain se ha convertido en una nueva frontera de capitales de riesgo que ha atraído la atención de bancos, gobiernos y otras corporaciones comerciales. Blockchain está a punto de convertirse en la invención más emocionante después de Internet; mientras que el segundo conecta el mundo a habilitar nuevos modelos comerciales basados en procesos en línea, el primero ayuda a resolver el problema de confianza de manera más eficiente a través de la computación en red (Zhao, Fan, \& Yan, 2016). La tecnología blockchain que sustenta a monedas digitales como el Bitcoin, podría tener consecuencias de largo alcance para todos los aspectos de la sociedad moderna. La tecnología Blockchain es esencialmente una base de datos de activos que se pueden compartir a través de una red de múltiples sitios, geografías o instituciones. Además Blockchain tiene la capacidad de ejecutar scripts autónomos. Este es el concepto de contrato inteligente; código controlado por datos que pueden representar una aplicación lógica verificable y ayuda a automatizar un conjunto de reglas del sistema (Huckle, Bhattacharya, White, \& Beloff, 2016).

Para abordar los problemas de la adopción de blockchain en un entorno empresarial, son tres los niveles de estudios que pueden ser llevados a cabo por investigadores, incluidos los conceptuales, prescriptivos, y niveles descriptivos. Actualmente, la investigación comercial en blockchain se encuentra principalmente en el nivel conceptual que conceptualizan las innovaciones de blockchain en negocios y en el nivel prescriptivo 
que describe las aplicaciones comerciales de la cadena. La investigación en el nivel descriptivo está rezagada, ya que se necesitan más esfuerzos para descubrir nuevas explicaciones y teorías subyacentes al fenómeno blockchain. Por ejemplo, hay una necesidad de determinar la validez económica y social de las aplicaciones blockchain. La investigación de Blockchain proviene de varias disciplinas relevantes, tal como científicas, comerciales y aplicaciones tecnológicas. Inicialmente, la investigación en blockchain comienza con técnicas y problemas de negocios, y se involucrarán más disciplinas a medida que los impactos de blockchain penetren profundamente en la sociedad y los gobiernos (Zhao, Fan, \& Yan, 2016).

Blockchain es un ledger distribuido creado por bloques que contienen detalles de transacciones conectados en orden cronológico para formar una serie de cadena. Es un libro mayor distribuido en el cual los participantes de la red peer- to-peer (P2P) de Blockchain, y no el administrador central, genera bloques. Las posibilidades de uso de Blockchain son reconocidas en muchos campos diferentes, lo que resulta en muchos desarrollos y estudios que se llevan a cabo, y las inversiones están sucediendo activamente. Blockchain es una tecnología para asegurar la integridad y confiabilidad de los registros de transacciones sin tercero como proveedor de servicios de confianza, haciendo que todos los participantes de la red creen, graben, almacenen y verifiquen la información de la transacción conjuntamente, y tiene la estructura para realizar diversos servicios de aplicaciones basados en infraestructura de red distribuida, utilizando tecnologías de seguridad incluyendo Hash, firma digital y criptografía. La tecnología Blockchain fue diseñada para guardar y usar una criptomoneda llamada Bitcoin de manera segura. (Zhao, Fan, \& Yan, 2016).

Blockchain es un sistema que permite escribir movimientos de tokens en un gran libro virtual que funciona a modo de libro de contabilidad para una moneda. Este libro ha demostrado ser intocable, gracias a estar completamente distribuido y constantemente actualizado con las nuevas entradas contables que se producen. Las entradas contables se agrupan por bloques antes de ser escritas en gran libro de contabilidad que es el blockchain. De esta manera blockchain pasa a ser un gran libro de contabilidad que puede ser escrito por cualquier entidad, pero una vez escrito no puede ser modificado, aunque cualquiera puede leerlo (Lérida \& Pérez, 2016). 
Una amplia gama de activos escasos pueden ser representados por los tokens de Blockchain, como monedas, valores, propiedades, certificados, entre otros. Los tokens de Blockchain generalmente tienen un suministro fijo o siguen un programa de suministro transparente, lo que los hace antiinflacionarios. Además, pueden transferirse entre partes sin la participación de una entidad central y pueden negociarse en intercambios de divisas digitales sin fronteras. Hay dos tipos principales de tokens de blockchain: moneda y token.

Una moneda suele ser nativa de una cadena de bloques. Dado que la tecnología blockchain se basa en la criptografía, dicha moneda generalmente se denomina criptomoneda. El ejemplo principal es Bitcoin, que es la moneda nativa de la cadena de bloques de Bitcoin (Nakamoto, 2008). El blockchain de Bitcoin permite a los usuarios almacenar y transferir bitcoins en una red de igual a igual. Otro ejemplo es Ether, que es la moneda nativa de la cadena de bloques de Ethereum. Al igual que la cadena de bloques de Bitcoin, la cadena de bloques Ethereum permite a los usuarios almacenar y transferir Ethers en una red Peer-to-Peer (P2P). Además, el blockchain de Ethereum puede potenciar contratos inteligentes y aplicaciones descentralizadas, que deben usar Ether para pagar los servicios computacionales proporcionados por la plataforma Ethereum (Chen \& Yan, 2018).

A diferencia de una moneda, un token no es nativo de una cadena de bloques, sino que se crea sobre un blockchain y se rige por un contrato inteligente. En la plataforma Ethereum, por ejemplo, la mayoría de los tokens se rigen por contratos inteligentes siguiendo el estándar común llamado ERC20, que especifica un conjunto de funciones y eventos que deben implementar todos los contratos inteligentes que cumplan con ERC20. Siguiendo el mismo estándar, los tokens ERC20 se pueden reconocer y comprender fácilmente en el ecosistema de Ethereum. Los tokens ERC20 se han creado para representar una amplia gama de activos digitalizados, lo que les permite desempeñar papeles importantes en el ecosistema de Ethereum. El valor de estos tokens depende del valor de los activos y servicios subyacentes que representan estos tokens. Por ejemplo, TenX (2017) es un servicio de pago habilitado con blockchain; tiene una billetera que permite a los usuarios almacenar sus criptomonedas y una tarjeta que permite a los usuarios gastarlas. TenX ha emitido el token de pago. Los usuarios de la tarjeta TenX 
pueden ganar el token de pago, al igual que los usuarios de tarjetas de crédito pueden ganar puntos de recompensa (Chen \& Yan, 2018).

\subsection{Tipos de blockchain}

Los tipos de blockchain se pueden clasificar en función del acceso a los datos, la

distinción entre los tipos de blockchain es el esquema del libro distribuido y quién puede participar en el sistema (Viriyasitavat \& Hoonsopon, 2018). De esta manera tenemos blockchain públicas, privadas e hibridas.

Blockchain Públicas: son de tipo abierto, en el que cualquiera puede participar. Todos los participantes pueden acceder libremente a datos y realizar transacciones, pero dado que numerosos usuarios no verificados están participando, se necesita cifrado y verificación avanzadas, y por lo tanto, la expansión de la red se torna lenta y difícil. Además, el blockchain público forma una perfecta estructura distribuida, y los participantes de la red son pseudoanónimos, por lo tanto no es apropiado para los servicios financieros que necesitan ser controlados por la información centralizada de los sistema de gestión (Oh \& Shong, 2017). También permiten que cualquiera acceda y mantenga el libro mayor distribuido con permisos para validar la integridad ejecutando un mecanismo de consenso. Una red pública de Blockchain está completamente abierta y distribuida; cualquiera puede unirse, participar y abandonar el sistema libremente. Por lo tanto, este sistema opera bajo nodos desconocidos y no confiables (Viriyasitavat \& Hoonsopon, 2018).

Blockchain Privadas: en ella el propietario genera y maneja el Blockchain. Esto es apropiado si el propietario desea administrar la Blockchain como el sistema centralizado (Oh \& Shong, 2017). Los libros contables son compartidos y validados por un grupo predefinido de nodos. El sistema requiere iniciación o validación a los nodos que desean ser parte del sistema. Los nodos autorizados son responsables de mantener el consenso. Blockchain privadas son adecuadas para sistemas cerrados, donde todos los nodos son completamente confiables. En definitiva es el propietario quien tiene la máxima autoridad para controlar el acceso a nodos autorizados (Viriyasitavat \& Hoonsopon, 2018).

Blockchain Hibridas (Consorcio): es el tipo intermedio de blockchain pública y privada. A diferencia de Blockchain Privadas en el que el propietario tiene la autoridad, 
son los nodos preestablecidos quienes tienen la autoridad en este tipo de Blockchain. Por lo tanto, Blockchain Hibridas mantienen una estructura distribuida al mismo tiempo que fortalece la seguridad mediante una participación limitada, y resuelve el problema de la lenta velocidad de transacción y los problemas de escalabilidad de la red planteados en Blockchain Pública. Por lo tanto, Blockchain Hibridas podrían ser utilizadas para transacciones entre instituciones financieras (Oh \& Shong, 2017).

La Blockchain hibrida es adecuada para sistemas semicerrados compuestos por unas pocas empresas, a menudo organizadas en forma de consorcio. El grado de apertura de los datos varía, por lo general con controles de acceso, definidos por el consorcio, para controlar el acceso en ambos participantes y la información dentro de Blockchains. A pesar de que el sistema no está completamente abierto, los beneficios de la descentralización se pueden obtener parcialmente. Hyperledger Fabric, Ripple y Stellar son ejemplos de implementaciones de Blockchain Hibridas (Viriyasitavat \& Hoonsopon, 2018).

Por lo tanto, las instituciones financieras están prestando atención a Blockchain Hibridas y Privadas que aprovecharán al máximo ventajas de Blockchain, como la reducción de costos, sin perder la autoridad de control del sistema e iniciativa, que son necesarios en el servicio financiero.

Según (Viriyasitavat \& Hoonsopon, 2018), todos los tipos de Blockchain comparten las siguientes similitudes con respecto a los beneficios que ofrece esta tecnología:

- Operan en una red P2P que proporciona cierto grado de descentralización,

- Múltiples nodos mantienen la integridad del libro mayor a través de mecanismos de consenso,

- Los datos se almacenan en Blockchain que proporciona inmutabilidad, incluso cuando algunos nodos son defectuosos o maliciosos. 
Tabla 1. Características según tipo de blockchain

\begin{tabular}{|c|c|c|c|}
\hline $\begin{array}{l}\text { Tipos de } \\
\text { Blockchain }\end{array}$ & Blockchain Publica & Blockchain Híbrida & Blockchain Privada \\
\hline Entidad gestora & $\begin{array}{l}\text { Todos los participantes } \\
\text { (descentralización) }\end{array}$ & $\begin{array}{l}\text { Participantes que } \\
\text { pertenezcan al consorcio. }\end{array}$ & $\begin{array}{l}\text { Una institución central tiene } \\
\text { toda la autoridad. }\end{array}$ \\
\hline Gobernanza & $\begin{array}{l}\text { Es muy difícil cambiar la regla } \\
\text { que se ha hecho. }\end{array}$ & $\begin{array}{l}\text { Las reglas podrian } \\
\text { cambiarse con relativa } \\
\text { facilidad de acuerdo con el } \\
\text { acuerdo entre los } \\
\text { participantes del consorcio. }\end{array}$ & $\begin{array}{l}\text { Las reglas podrian cambiarse } \\
\text { fácilmente de acuerdo con la } \\
\text { decisión tomada por la } \\
\text { institución central. }\end{array}$ \\
\hline $\begin{array}{l}\text { Velocidad de } \\
\text { transacción }\end{array}$ & $\begin{array}{l}\text { Es difícil expandir la red y la } \\
\text { velocidad de transacción es } \\
\text { lenta. }\end{array}$ & $\begin{array}{l}\text { Es fácil expandir la red y la } \\
\text { velocidad de transacción es } \\
\text { rápida. }\end{array}$ & $\begin{array}{l}\text { Es muy fácil ampliar la red y } \\
\text { la velocidad de transacción es } \\
\text { rápida. }\end{array}$ \\
\hline Acceso a los datos & Cualquiera puede acceder & $\begin{array}{l}\text { Solo usuarios autorizados } \\
\text { pueden acceder }\end{array}$ & $\begin{array}{l}\text { Solo usuarios autorizados } \\
\text { pueden acceder }\end{array}$ \\
\hline Identificabilidad & Seudo-anónimo & Identificable & Identificable \\
\hline $\begin{array}{l}\text { Prueba de } \\
\text { transacción }\end{array}$ & $\begin{array}{l}\text { La entidad para la prueba de la } \\
\text { transacción se decide } \\
\text { mediante algoritmos como } \\
\text { PoW y PoS, y no se puede } \\
\text { conocer de antemano. }\end{array}$ & $\begin{array}{l}\text { La entidad para la prueba de } \\
\text { la transacción se conoce a } \\
\text { través de la autenticación, y } \\
\text { la verificación de la } \\
\text { transacción y generación de } \\
\text { bloques se realizan de } \\
\text { acuerdo con las reglas } \\
\text { acordadas de antemano. }\end{array}$ & $\begin{array}{l}\text { La prueba de transacción es } \\
\text { realizada por la institución } \\
\text { central. }\end{array}$ \\
\hline $\begin{array}{l}\text { Casos de } \\
\text { utilización }\end{array}$ & Bitcoin & R3CEV & $\begin{array}{l}\text { Linq, una plataforma de } \\
\text { mercado bursátil para } \\
\text { compañias sin cotización } \\
\text { NASDAQ }\end{array}$ \\
\hline
\end{tabular}

Fuente: Oh \& Shong (2017)

A diferencia de Blockchain Publicas, que proporciona pseudoanonimato, es

posible identificar el sujeto en Blockchain Privadas. Las transacciones se manejan rápidamente, la expansión de la red es fácil y podría ser modificado de la manera que el usuario desee, y por lo tanto, es adecuado para servicios financieros. Por lo tanto, está recibiendo atención de las compañías e instituciones (Oh \& Shong, 2017). El Cuadro $\mathrm{N}^{\circ} 1$, presenta las características de cada tipo de blockchain.

\subsection{Innovación del Dapp blockchain}

La tecnología blockchain como base para libro mayor distribuidos ofrece una innovadora plataforma para un nuevo mecanismo de transacciones descentralizado y transparente en industrias y negocios. Las características heredadas de esta tecnología mejoran la confianza a través de la transparencia y la trazabilidad dentro de cualquier 
transacción de datos, bienes y recursos financieros. A pesar de las dudas iniciales sobre esta tecnología, Recientemente, los gobiernos y las grandes corporaciones han investigado para adoptar y mejorar esta tecnología en varios dominios de aplicaciones, desde las finanzas, las industrias sociales y legales, hasta el diseño, fabricación y redes de la cadena de suministro (Abeyratne \& Monfared, 2016).

A grandes rasgos, lo realmente innovador de blockchain es que su sistema permite escribir los movimientos de tokens (por ejemplo bitcoins) en un gran libro virtual que funciona a modo de gran fichero de contabilidad para una moneda. Ese libro ha demostrado ser inatacable, y se basa en estar completamente distribuido y ser actualizado constantemente con las nuevas entradas contables que se van produciendo. Esas entradas contables se agrupan por bloques antes de escribirse en el gran libro de contabilidad que es el blockchain. Es decir, blockchain es una especia de gran libro de contabilidad que puede ser escrito por cualquier entidad, pero que una vez escrito no hay forma de modificarlo, aunque cualquiera puede leerlo.

La unión de los bloques en los que se agrupan los apuntes de contabilidad es lo que se conoce como cadena de bloques o blockchain. Es decir, el blockchain es un gran libro de contabilidad que se va incrementando conforme se van produciendo movimientos y que se caracteriza porque una vez que se crea un movimiento de tokens y se inscriben, este movimiento nunca podrá ser modificado por nadie, lo que le da legitimidad y la posibilidad de gestionar transacciones entre personas que no se conocen a través de redes que son inseguras originalmente.

La primera criptomoneda exitosa Bitcoin se basó en la tecnología blockchain. Y desde entonces se sabe que el blockchain es una base de datos distribuida para todas las transacciones y resuelve el problema del doble gasto combinando la tecnología peer-topeer con criptografía de clave pública. Literalmente, un blockchain es una cadena de bloques de información que registra las transacciones de Bitcoin. Los algoritmos de creación y la infraestructura computacional, para insertar y usar los bloques se consideran como la tecnología blockchain. La característica clave principal de la tecnología blockchain es la capacidad de rastrear transacciones dentro de bases de datos públicas descentralizadas y, por lo tanto, excluye la falsificación y el fraude. La esencia de blockchain radica en su capacidad para admitir transacciones confiables a través de redes 
computacionales en lugar de supervisión y control humano. El consenso distribuido y el anonimato son dos importantes características de la tecnología blockchain. Una serie de grandes compañías industriales, como IBM, Microsoft, Intel y NEC invierten actualmente en la explotación de blockchain para enriquecer su cartera de productos (Efanov \& Roschin, 2018).

Blockchain proporciona intrínsecamente varias ventajas tecnológicas clave para los usuarios que son implicaciones de su arquitectura estructural. Algunos de los cuales incluyen durabilidad, transparencia, inmutabilidad e integridad del proceso (Abeyratne \& Monfared, 2016):

- Durabilidad: las redes descentralizadas eliminan puntos únicos de falla en lugar de sistemas centralizados. Esta distribución del riesgo entre sus nodos hace que las cadenas de bloques sean mucho más duraderas que los sistemas centralizados y que sean más adecuadas para evitar los ataques maliciosos.

- Transparencia: cada nodo en la red mantiene una copia idéntica de una cadena de bloques, lo que permite la auditoría e inspección de los conjuntos de datos en tiempo real. Este nivel de transparencia hace que las actividades y operaciones de la red sean muy visibles, reduciendo así la necesidad de confianza

- Inmutabilidad: los datos que se almacenan en un blockchain público distribuido son prácticamente inmutables debido a la necesidad de validación por parte de otros nodos y rastreabilidad de cambios. Esto permite a los usuarios operar con el mayor grado de confianza, ya que la cadena de datos es exacta e inalterable.

- Integridad del proceso: los protocolos distribuidos de código abierto se ejecutan por naturaleza exactamente como están escritos en el código. Los usuarios pueden estar seguros que las acciones descritas en el protocolo se ejecutan de forma correcta y oportuna sin necesidad de intervención humana.

Sin embargo, esta tecnología requiere cierta infraestructura de TI para todos los actores, como el acceso a Internet, que puede ser poco práctico en este momento para algunos proveedores remotos de materia prima. Los perfiles digitales tendrían que mantenerse actualizados constantemente a través de sistemas manuales o automatizados, como etiquetas simples o RFID. Los contratos inteligentes deben implementarse y ser 
incrustado en el sistema para proporcionar incentivos que permitan a blockchain controlar el progreso de un proceso de negocios. En adición, la capacidad de desempeño de dicho sistema puede ser un cuello de botella para la implementación de la solución propuesta (Abeyratne \& Monfared, 2016).

Tabla 2. Ventajas y desventajas según características de blockchain

Fuente: Oh \& Shong (2017).

Desde el descubrimiento de la tecnología blockchain, este campo ha experimentado un crecimiento masivo a través de varios conceptos innovadores y tecnológicos. Inicialmente, esta tecnología ganó una reputación negativa debido a su asociación con compras imposibles de rastrear en la 'red oscura' donde los usuarios usarían monedas digitales como Bitcoin para realizar compras de forma anónima. Sin embargo, en los últimos años, muchas grandes empresas como IBM, JPMorgan y Barclays han invertido en investigación y desarrollo de la tecnología Blockchain. Debido a tal interés de las principales organizaciones, y la gran cantidad de dinero circulando en criptomonedas, empresarios, organizaciones innovadoras y creativas han sido atraídos por este nuevo campo de la tecnología de la información. Este rápido crecimiento en el campo ha cambiado la perspectiva de muchos gobiernos para ver el potencial de esta tecnología por sobre su relación inicial con actividades ilegales (Abeyratne \& Monfared, 2016). El interés corporativo, industrial y gubernamental en las tecnologías Blockchain ha crecido considerablemente porque las aplicaciones se extienden mucho más allá del dominio de las criptomonedas.

Según Swan (2018) existen cuatro clases principales de aplicación para la tecnología Blockchain:

1. Activos monetarios (moneda, pagos, remesas, finanzas, valores e instrumentos financieros),

2. Propiedad (terrenos, bienes raíces y registros de títulos de auto),

3. Contratos (acuerdos comerciales, licencias, registro, testamentos $\mathrm{y}$ fideicomisos, acuerdos de asociación y registro de propiedad intelectual) 
4. Credenciales de identidad (pasaporte, visa, licencia de conducir y registros de nacimiento).

Para que una aplicación se considere una DApp, debe cumplir con los siguientes criterios:

- La aplicación debe ser 100\% código abierto,

- La aplicación debe funcionar de forma autónoma, sin entidad que controle la mayor parte de sus tokens,

- La aplicación adopta su protocolo en respuesta a las mejores propuestas y todo cambio debe ser decidido por consenso de sus usuarios antes de hacerse efectivas,

- Datos y registros de funcionamiento de la aplicación deben ser almacenados criptográficamente por medio de una blockchain publica, añadiendo transparencia y seguridad como cualidades de la aplicación descentralizada,

- Las operaciones realizadas deben ser almacenadas en bloques por lo que deben ser verificadas mediante protocolo basado en algoritmo prueba de trabajo (PoW) o prueba de participación (PoS),

- Si la DApp funciona según algoritmo criptográfico (PoW o PoS) para la validación de sus bloques, significa que existen mineros o validadores que realizan ese trabajo. Dichos mineros deben ser recompensados por el trabajo realizado mediante criptomonedas o tokens.

- Por validar bloques en la DApp se generan criptomonedas o tokens, con ellas serán recompensados los mineros y además servirá para intercambiar valor dentro de la aplicación.

Tras conocer las características que tiene que tener una DApp para considerarse como tal, pasaremos a diferenciar los distintos tipos de DApps que existen. Esta clasificación de las aplicaciones descentralizadas depende de si poseen su propia blockchain o si bien utilizan la cadena de bloques de otra DApp.

Según este criterio podríamos diferenciar tres tipos de DApps en función del uso que hagan de sus tokens y del blockchain al que estén asociadas: 
Para que una aplicación se considere una DApp, debe cumplir con los siguientes criterios:

- La aplicación debe ser 100\% código abierto,

- La aplicación debe funcionar de forma autónoma, sin entidad que controle la mayor parte de sus tokens ,

- La aplicación adopta su protocolo en respuesta a las mejores propuestas y todo cambio debe ser decidido por consenso de sus usuarios antes de hacerse efectivas,

- Datos y registros de funcionamiento de la aplicación deben ser almacenados criptográficamente por medio de una blockchain publica, añadiendo transparencia y seguridad como cualidades de la aplicación descentralizada,

- Las operaciones realizadas deben ser almacenadas en bloques por lo que deben ser verificadas mediante protocolo basado en algoritmo prueba de trabajo (PoW) o prueba de participación $(\mathrm{PoS})$,

- Si la DApp funciona según algoritmo criptográfico (PoW o PoS) para la validación de sus bloques, significa que existen mineros o validadores que realizan ese trabajo. Dichos mineros deben ser recompensados por el trabajo realizado mediante criptomonedas o tokens.

- Por validar bloques en la DApp se generan criptomonedas o tokens, con ellas serán recompensados los mineros y además servirá para intercambiar valor dentro de la aplicación.

Tras conocer las características que tiene que tener una DApp para considerarse como tal, pasaremos a diferenciar los distintos tipos de DApps que existen. Esta clasificación de las aplicaciones descentralizadas depende de si poseen su propia blockchain o si bien utilizan la cadena de bloques de otra DApp.

Según este criterio podríamos diferenciar tres tipos de DApps en función del uso que hagan de sus tokens y del blockchain al que estén asociadas:

Tipo I.- Poseen su propia blockchain como es el caso de Bitcoin, Ethereum y Litecoin. Una DApp de tipo I es el equivalente a un sistema operativo de un ordenador. 
- Tipo II.- Utilizan blockchains de una DApp de tipo I. Estas DApps son protocolos $\mathrm{y}$ tienen sus propios tokens necesarios para su funcionamiento, pero no tienen una blockchain propia. Por tanto se realiza una equivalencia entre el token de la DApps tipo I sobre la que funcionan y sus propios tokens. Una DApp del tipo II corresponde al equivalente de un programa con un propósito general (Ej: Word, Excel, etc.).

- Tipo III.- Utilizan el protocolo de una aplicación descentralizada tipo II. También son protocolos y utilizan tokens propios que son necesarios para su función, pero no actúan directamente sobre las de tipo I sino que utilizan funciones de tipo II que permiten un desarrollo más rápido y sencillo. El símil de una DApp tipo III es un software especializado, una herramienta que agrega un servicio específico a un programa (Ej: Crystal Ball para Excel).

\section{Conclusiones}

Actualmente la tecnología blockchain se encuentra en etapa de crecimiento

apoyado por grandes empresas que apuestan por su desarrollo y potenciales usos. Existen una variedad de industrias en el ámbito privado que ven a la tecnología blockchain como una forma de mejorar sus procesos reduciendo tiempos de procesos, reducción de costos, aumento de la seguridad, transparencia y estabilidad del sistema. Sin embargo el verdadero potencial de la tecnología se puede apreciar en una red mayor mediante una rápida adopción, con la generación de nuevos modelos de negocio mediante aplicaciones públicas descentralizadas apoyando el desarrollo de la infraestructura en un aspecto económico, empresarial y social. Sin embargo, la adopción requiere de la articulación tanto del ámbito tecnológico como político, social y económico para llegar a una fase de maduración, si hoy existe un ecosistema robusto aun falta el desarrollo en temas relacionados a la regulación y al conocimiento por parte del macroentorno.

El análisis de la tecnología blockchain requiere de la comprensión total de su

ecosistema, entendiendo el aporte generado a través de la cadena de valor de la industria por cada uno de los actores de acuerdo a sus modelos de negocios. Esto permite evaluar posibilidades de generación de nuevos modelos de negocios, reestructuración de los existentes para las empresas que serán impactadas y la creación de nuevos negocios que utilicen el desarrollo actual de la tecnología. 
Si bien en la actualidad la tecnología blockchain está siendo adoptada

principalmente por la industria financiera, para la mejora de sus procesos y sus servicios, también es factible su utilización en otros sectores del ámbito privado como cadena de suministro, legal, salud, social, educación, energía y sustentabilidad. Sin embargo son las empresas que basan sus modelos de negocio en el registro e intercambio de información y valor mediante cualquier clase de activos las que poseen un mayor riesgo de ser impactadas económica y financieramente por la tecnología blockchain, por medio de las aplicaciones descentralizadas que buscan el beneficio de todos sus miembros. Este tipo de empresas deben buscar formas de reducir las amenazas de la tecnología ya sea incorporándola o ajustando sus modelos de negocio.

El objetivo general de la tesina es conocer el entorno de negocios de la tecnología

blockchain, así como sus usos, repercusiones y beneficios para los negocios. Como el entorno de blockchain posee una gran cantidad de actores relevantes, el análisis mediante al ecosistema propuesto y la descripción de los modelos de negocios de sus participantes permiten entender cómo se agrega, entrega y captura valor a través de la cadena de valor de la industria creando modelos negocio o modificando los existentes. De esta manera, tenemos que el sector privado actualmente adopta la tecnología blockchain de tipo privada y consorcio para la mejora de sus procesos beneficiando su captura de valor, mientras la blockchain pública es utilizada mediante aplicaciones descentralizadas para construir modelos de negocios confiables, transparentes que distribuyen sus beneficios con la comunidad agregando y entregando valor.

\section{Referencias}

Abeyratne, S. A., \& Monfared, R. P. (2016). Blockchain Ready Manufacturing Supply Chain Using Distributed Ledger. International Journal of Research in Engineering and Technology, 5, 1-10.

Bermúdez, A. M. (2016). Estudio de la utilización de protocolos blockchain en sistemas de votación electrónica. Barcelona.

Caballero, J. (2018). Estudio de tecnologías Bitcoin y Blockchain. Tesis, Universidad Oberta de Catalunya. 
Carson, B., Romanelli, G., Walsh, P., \& Zhumaev, A. (Junio de 2018). Mckinsey\&Company. Obtenido de https://www.mckinsey.com/businessfunctions/digital-mckinsey/ourinsights/blockchain-beyond-the-hype-what- is-thestrategic-business-value

Chen, \& Yan. (2018). Blockchain tokens and the potential democratization of entrepreneurship and innovation. Business Horizons, Volume 61, Issue 4, 567-575.

Chen, Y. (2018). Blockchain tokens and the potential democratization of entrepreneurship and innovation. Business Horizons, Volume 61, Issue 4, 567-575.

Chesbrough, H. (2010). Business Model Innovation: Opportunities and Barriers. Long Range Planning, 43, 354-363.

Efanov, D., \& Roschin, P. (2018). The All-Pervasiveness of the Blockchain Technology. Procedia Computer Science, 123, 116-121.

Ethereum. (2018). Obtenido de https://miethereum.com/smart- contracts/dapps/\#toc16

Forbes, I. (Noviembre de 2016). Forbes Insights.

Obtenido de

https://www.forbes.com/forbesinsights/sap_transactions/index.html

Gimeno, J. C. (2018). Estudio de tecnologías Bitcoin y Blockchain. Obtenido de http://hdl.handle.net/10609/81268

Gönenç Gürkaynak, I. Y. (2018). Intellectual property law and practice in the blockchain realm. Computer Law \& Security Review, 34, 847-862.

Gordon, W. J., \& Catalini, C. (2018). Blockchain Technology for Healthcare: Facilitating the Transition to Patient-Driven Interoperability. Computational and Estructural Biotechnology Journal, 16, 224-230.

Huckle, S., Bhattacharya, R., White, M., \& Beloff, N. (2016). Internet of thing; Blockchain and Share Economy Applications. Procedia Computer Science, 98, 461-466.

Hwang, J., Choi, M.-i., Lee, T., Jeon, S., Kim, S., Park, S., \& Park, S. (Diciembre de 2017). Energy Prosumer Business Model Using Blockchain System to Ensure Transparency and Safety. Energy Procedia, 141, 194-198. 
Kshetri, N. (2018). Blockchain's roles in meeting key supply chain management objectives. International Journal of Information Management, 39, 80-89.

Lérida, J. L., \& Pérez, J. J. (2016). La economía de Blockchain - Los modelos de negocio de la nueva web. Creative Commons.

Macías, M. (11 de marzo de 2012). Advenio Strategy\&Business and Design. Obtenido de http://advenio.es/crea-tu-business-model-canvas-con- google-docs/

Melanie Swan. (2018). Blockchain for Business: Next-Generation Enterprise Artificial Intelligence Systems. Advances in Computers, 11, 121-162.

Meunier, S. (2018). Blockchain 101: What is Blockchain and How Does This Revolutionary Technology Work? Transforming Climate Finance and Green Investment with Blockchains., 23-34.

miethereum. (2018). Ethereum. Obtenido de

https://miethereum.com/smart-contracts/dapps/\#toc1 miethereum. (29 de Agosto de 2014). Obtenido de https://miethereum.com/mineria/

Millard, C. (2018). Blockchain and law: Incompatible codes? Computer Law \& Security Review, 34, 843-846.

Najmaei, A. (2015). Business Model Value Creation, Value Capture, and Information Technologies. Encyclopedia of Information Science and Technology, Third Edition, 549-557.

Nakamoto, S. (2008). Bitcoin: A Peer-to-Peer Electronic Cash System. Obtenido de https://bitcoin.org/es/

Nasdaq. (2016). Bussines Nasdaq. Obtenido de

https://business.nasdaq.com/Docs/Blockchain\%20Report\%20March\%20

2016_tcm5044-26461.pdf

Oh, J., \& Shong, I. (2017). A case study on business model innovations using Blockchain: focusing on financial institutions. Asia Pacific Journal of Innovation and Entrepreneurship, 11, 335-344.

Osterwalder, A., \& Pigneur, Y. (2010). Business Model Generation. New Jersey: John Wiley \& Sons, Inc. 
Porxas, N., \& Conejero, M. (2018). Tecnología blockchain: funcionamiento, aplicaciones y retos jurídicos relacionados. Actualidad Jurídica Uría Menéndez, 48, 24-36.

Preukschat, A. (2017). Blockchain la revolución industrial de internet. Paidos Empresas.

Reyna, A., Martín, C., Chen, J., Soler, E., \& Díaz, M. (2018). On blockchain and its integration with IoT. Challenges and opportunities. Future Generation Computer Systems, 88, 173190.

Riasanow, T., Burckhardt, F., Setzke, D. S., Böhm, M., \& Krcmar, H. (2018). The Generic Blockchain Ecosystem and its Strategic Implications. Twenty- fourth Americas Conference on Information Systems, 1-10.

Santiago, I. (2016). La Revolución de la Tecnología de las Cadenas de Bloques y su Impacto. Sevilla. Obtenido de https://ssrn.com/abstract=2846816

Sanz, J. M. (2017). Blockchain per l'educació. Barcelona. Obtenido de https://ddd.uab.cat/record/181567

Supranee, S., \& Rotchanakitumnuai, S. (2017). The Acceptance of the Application of Blockchain Technology in the Supply Chain Process of the Thai Automotive Industry.

Proceedings of The 17th International Conference on Electronic Business, 30, 252-257.

Swan, M. (2015). Blockchain Blueprint for a New Economy. O’Reilly .

Teece, D. J., \& Linden, G. (2017). Business models, value capture, and the digital enterprise. Journal of Organization Design, 1-14.

Viriyasitavat, W., \& Hoonsopon, D. (2018). Blockchain characteristics and consensus in modern business processes. Journal of Industrial Information Integration.

Zhang, P., Schmidt, D. C., \& Jules White, G. L. (2018). Blockchain Technology Use Cases in Healthcare. Advances in Computers, 111, 1-41.

Zhao, J. L., Fan, S., \& Yan, J. (2016). Overview of business innovations and research opportunities in blockchain and introduction to the special issue. Financial Innovation, 2, 1-7. 